\title{
Modeling and analyzing users' behavioral strategies with co-evolutionary process
}

\author{
Yutaro Miura ${ }^{1}$, Fujio Toriumi ${ }^{2}$ and Toshiharu Sugawara ${ }^{1 *}$ (B)
}

\section{*Correspondence:}

sugawara@waseda.jp

${ }^{1}$ Department

of Computer Science

and Communications

Engineering, Waseda

University, 3-4-1 Okubo,

Shinjuku, Tokyo 169-8555,

Japan

Full list of author information

is available at the end of the

article

\begin{abstract}
Social networking services (SNSs) are constantly used by a large number of people with various motivations and intentions depending on their social relationships and purposes, and thus, resulting in diverse strategies of posting/consuming content on SNSs. Therefore, it is important to understand the differences of the individual strategies depending on their network locations and surroundings. For this purpose, by using a game-theoretical model of users called agents and proposing a co-evolutionary algorithm called multiple-world genetic algorithm to evolve diverse strategy for each user, we investigated the differences in individual strategies and compared the results in artificial networks and those of the Facebook ego network. From our experiments, we found that agents did not select the free rider strategy, which means that just reading the articles and comments posted by other users, in the Facebook network, although this strategy is usually cost-effective and usually appeared in the artificial networks. We also found that the agents who mainly comment on posted articles/ comments and rarely post their own articles appear in the Facebook network but do not appear in the connecting nearest-neighbor networks, although we think that this kind of user actually exists in real-world SNSs. Our experimental simulation also revealed that the number of friends was a crucial factor to identify users' strategies on SNSs through the analysis of the impact of the differences in the reward for a comment on various ego networks.
\end{abstract}

Keywords: Social networking services, Agent-based simulation, Public goods game, Co-evolution, Facebook

\section{Background}

Nobody doubts that social networking services (SNSs), such as Twitter, Facebook, Instagram, and LinkedIn, have played an important role in our lives even though they have been developed in virtual places. There are various ways of using SNSs such as private and local communication, marketing, advertisements, and political campaigns. Unlike conventional mass media such as television, newspaper, and radio, SNSs are maintained by a large amount of content that is generated by users' voluntary participations; thus, SNSs collapse if too little content is posted on them. Due to this SNS characteristics, users can be lurkers or free riders who read content without posting any content because posting articles and comments imposes some costs on users. It is crucial to understand

(c) The Author(s) 2021. This article is licensed under a Creative Commons Attribution 4.0 International License, which permits use, sharing, adaptation, distribution and reproduction in any medium or format, as long as you give appropriate credit to the original author(s) and the source, provide a link to the Creative Commons licence, and indicate if changes were made. The images or other third party material in this article are included in the article's Creative Commons licence, unless indicated otherwise in a credit line to the material. If material is not included in the article's Creative Commons licence and your intended use is not permitted by statutory regulation or exceeds the permitted use, you will need to obtain permission directly from the copyright holder. To view a copy of this licence, visit http://creativeco mmons.org/licenses/by/4.0/. 
why users voluntarily post content so often while others behave as free riders, and it is worth knowing the mechanism by which SNSs flourish and keep active.

There are many studies that discuss the interaction design methodologies and mechanisms for active SNSs from different viewpoints, such as analyzing exchange patterns [1], social and psychological viewpoints [2], evolutionary game theory [3], and network structures and information diffusion [4-6]. Our study has adopted an evolutionary game-theoretic method, which is a learning algorithm to understand users' reasonable behavioral strategies and what incentives affect users' strategies in SNSs. A few studies have attempted to understand incentives for voluntary participation on SNSs using game-theoretic models. Toriumi et al. [3], for example, have proposed a meta-rewards game that models users' interactions on SNSs and tried to identify their common behaviors using evolutionary computation. Because SNSs have a number of characteristics that can be observed in public-goods game, the meta-rewards game is an extension of Axelrod's meta-norms game [7]. Then, they analyzed what could prompt cooperation, i.e., continuing posting articles/comments, even in social dilemma situations. Their results show that cooperation on SNSs evolved by giving a meta reward, i.e., a reward as posting a comment on comments. Then, Hirahara et al. [8,9] proposed an SNSnorms game by adding the structural interaction features of SNSs to the meta-rewards game. For example, users who respond to comments on posted articles are usually those who posted the original articles. Then, they identified the optimal or shared reasonable behavior in this game from the experiments using agent-based simulations on artificial complex networks and a Facebook network.

We have to consider two issues of the conventional studies that used evolutionary algorithms for network analysis to search for a common better strategy for all users. First, applying genetic algorithms (GAs) to find a better or dominant strategy that is common for all users does not fit actual SNSs. For example, the appropriate strategy for hub users (such as celebrities) that have very many followers may not be advantageous for other ordinary users. All users probably seem homogeneous in the sense that they want to maximize the rewards received through the game. However, they are in diverse surroundings because the numbers of friends/followers are very different, and their appropriate behaviors must be affected by their surrounding users. Therefore, their behavioral strategies must be evolved by taking into account the strategies of the surrounding users. Second, the conventional studies focused on only the learned final strategies. We think that these strategies evolved through iterative interactions, so the process of learning must be more complicated. Thus, we need to attempt to analyze the process of learning; i.e., how they learned the strategies that mutually affected neighboring users.

This discussion motivated us to more carefully analyze the differences of the evolved strategies between in the artificial networks, such as the connecting nearest-neighbor networks, and in a natural network generated by Facebook and its ego networks by reflecting the diversity of agents' appropriate strategies in their surroundings. For this purpose, we propose to use the multiple-world genetic algorithm (multiple-world GA) [10, 11], which is a genetic algorithm of co-evolutionary computation [12] for evolutionary network analysis, to maintain the diversity of nodes in complex networks, where co-evolution is a phenomenon in which different species affect each other and evolve together. Although many studies have investigated how to avoid a local optimal solution and have 
proposed effective methods such as distributed GA [13] and diversity control oriented $G A$ [14] to find a better solution in a whole system, our objective is to maintain the individuality of each agent' strategy in the network and analyze a combination of their different strategies in a solution in social network analysis. In the multiple-world GA, a complex network including all nodes (agents) and edges are duplicated to several networks in which agents in the same position have different strategies. Then, all agents interact with their neighbors using various behavioral strategies in each copy of the network to simulate the effects of various strategies with the neighboring agents with different strategies. Therefore, agents can evolve their own diverse strategies that must be appropriate for the neighbors' various strategies. We have already analyzed the diverse strategies of the SNS-norms game in the complex networks of agents using the multipleworld GA [15]. However, this study used the artificial networks generated by the connecting nearest-neighbor model [16], but the natural networks may have characteristics different from those observed in the connecting nearest-neighbor networks.

Therefore, in this paper, we analyzed the characteristics of strategies evolved in the Facebook network as an example of a real-world network under the condition that all agents can evolve their own diverse strategies and compare them with those evolved in the connecting nearest-neighbor networks. Of course, the connecting nearest-neighbor networks have the network properties that are often observed in human society, but some features of the natural networks are still unknown; actually, we observed some interesting characteristics in the Facebook network that did not appear in the connecting nearest-neighbor networks. We also investigated the complex process of co-evolved strategies over the iterated game interactions and the distribution of strategies on the Facebook network.

\section{Related work}

Since communication using SNSs became an indispensable part of people's lives, many studies have investigated what factors affect users' motivation and the reasons they constantly use SNSs. Al-Debei et al. [17] examined the intentions of the users' continuous participation from social and behavioral perspectives, focusing on Facebook. Chen et al. [18] investigated four social factors: subjective norms, electronic word-of-mouth, images, and critical mass. They attempted to understand which factors affect their continuous use of social media. They then clarified that user satisfaction with social media applications is significantly affected by electronic word-of-mouth and effectively induces continuous use of social media. Lin and Lu [2] also empirically studied constant intention to use SNSs from the viewpoint of network externalities and motivation theory. They indicated that enjoyment is the most influential factor for users to motivate to join and keep using an SNS. They also conducted clustering analysis of gender and consequently revealed that the gender difference produces different influences.

Other studies have looked into why people become free riders or quit using SNSs [1921]. For example, Sun et al. [19] applied a motivation model to clarify why users in online communities stop providing content. The researches gave three factors that made users become free riders: long response delays, low response rates, sand low-quality messages. Then, they also insisted that external stimuli (a kind of reward) and new norms could encourage user activation on SNSs. Lörincz et al. [20] researched the key users who first 
left an SNS by giving a contrasting explanation based on the users' structural locations in the network. They claimed that a higher degree number (higher number of connections) and less clustered ego networks were effective in delaying early withdrawal from an SNS. Some researchers focused on the stress and exhaustion of the user when using SNSs [22, 23]. For example, Adeel et al. [22] utilized the stimulus-organism-response paradigm to empirically study the antecedents of intentions to discontinue using Facebook.

Abstract models of SNSs based on the game-theoretic approach have recently been proposed to investigate the characteristics of the SNSs from many aspects. Xiong et al. [24] proposed a lurker game and attempted to model the dilemma situations among the human behaviors observed in big SNSs. Deng et al. [25] proposed a theoretical dynamic model in social networks based on reciprocal altruism by utilizing the $N$-player prisoner's dilemma (NPD). There are very many game-theoretical studies on social networks and social media (e.g., [26-28]), and some of these studies insisted that online social networks have common features with public goods $[29,30]$. Therefore, some studies attempt to model SNSs in the framework of the evolutionary game like the public goods game [3, 9, 31, 32]. For example, Osaka et al. [31] extended the rewards game and meta-rewards game [3], which are the dual part of Axelrod's norms and meta-norms games, to include reciprocity between users and then clarified that the half-free rider is a suitable strategy for the continuous use of SNSs, where half-free riders are users who comment on the content posted by their close friends but behave as free riders for other content. Toriumi et al. [32] also extended the meta-rewards game to flexibly model users' behaviors on SNSs. Their simulation results indicate that providing content is encouraged more if potential commentators/responders can confirm that the content provider will respond to their comments.

However, these studies used the conventional GA to find the agents' evolved strategies under the assumption that the good strategies of neighboring agents are beneficial; this leads to strategies that have lost diversity by imitating other's strategies. Therefore, we focus more on the diversity of strategies that are the results of the individual network locations and the strategies of the neighboring agents.

\section{Modeling SNSs}

\section{Overview of multiple-world genetic algorithm (multiple-world GA)}

Here, we briefly explain the multiple-world GA; see Miura et al. [10, 11] for details. In the multiple-world GA, the network including all agents is duplicated to several networks where siblings, i.e., agents in the same position ${ }^{1}$, have different genes (parameters). Then, in each generation, all agents interact with the neighbors that are in identical locations in all duplicated networks but with different strategies, and then the agents for next the generation are generated based on the fitness values. Note that each duplicated network is often called world because the agents in each world have diverse experience by interacting with the same set of neighboring agents that behave differently.

Therefore, the process of the SNS-norms game [8] was slightly modified to adopt the multiple-world GA to find and examine diverse strategies that are reasonable for 
individual agents. The overview of this process consists of the following subprocesses (SPs).

SP 1: A number of copies (or worlds) of an agent network (a Facebook network in our experiments) are generated,

SP 2: Four rounds of an SNS-norms game are performed by individual agents in each world in one generation,

SP 3: Agents' strategies in each world are evolved for the next generation using the genes of sibling agents that had different experience, and

SP 4: We iterate SP 2 and SP 3 until the specified generations.

After this process, the genes of the original agents are defined as the gene of one sibling that earned the highest rewards; the details will be described later.

\section{Agent network}

In the SNS-norms game, agents that correspond to users on an SNS are nodes of a graph $G=(V, E)$, where $V=\left\{v_{1}, \ldots, v_{n}\right\}$ is the set of agents, and $E$ is the set of links that represent friend relationships between agents. Graph $G$ is also called the agent network. Agent $v_{i} \in V$ has a behavioral strategy denoted by two parameters $B_{i}$ and $L_{i}\left(0 \leq B_{i}, L_{i} \leq 1\right)$. Parameter $B_{i}$ is the probability of contributing to the SNS by posting an article. Parameter $L_{i}$ is the probability of giving a comment on a read article or a meta-comment, i.e. a comment that is a reply to a received comment. Therefore, agents with high $B_{i}$ and/ or $L_{i}$ actively participate on the SNS. Because our purpose in this paper is to investigate agents' behavior in an actual network, we focus on the agent network generated by Facebook (ego) networks from the Stanford Large Network Dataset Collection [33].

To start the SNS-norms game with the multiple-world GA, the master network $G=(V, E)$ is duplicated to $W$ networks for using the multiple-world GA, where a positive integer $W$ is the number of worlds. This process corresponds to the subprocess SP 1 in the previous subsection. For $1 \leq \forall l \leq W$, we represent the $l$-th network as $G^{l}=\left(V^{l}, E^{l}\right)$, where $V^{l}=\left\{v_{1}^{l}, \ldots, v_{n}^{l}\right\}$ is the set of agents in the $l$-th network, and $v_{i}^{l}$ is the copy of agent $v_{i} \in V$. For $\forall v_{i} \in V$, let $A_{i}=\left\{v_{i}^{1}, \ldots, v_{i}^{W}\right\}$ be the set of copy agents of $v_{i}$ in a different world, and the agents in $A_{i}$ are called the sibling agents or simply siblings. We also often call $v_{i}$ the master agent of $v_{i}^{l}$. Although the network characteristics of individual sibling agents on the duplicated networks are identical, initial strategies $B_{i}^{l}$ and $L_{i}^{l}$ are randomly given to all agents, so they can evolve differently, where the $B_{i}^{l}$ and $L_{i}^{l}$ parameters determine the strategy of $v_{i}^{l} \in V^{l}$. The strategies $B_{i}^{l}$ and $L_{i}^{l}$ in each duplicated network are encoded by three-bit genes for the genetic operators in the evolution phase; thus, they take on a discrete value, $0 / 7,1 / 7, \ldots$, or $7 / 7$.

\section{SNS-norms game}

All agents conduct three sequential steps in the SNS-norms game. The flow of the SNSnorms game is illustrated in Fig. 1, which shows the sequential steps of the SNS-norms game. Figure 2 shows the conceptual diagram of this game. For agent $v_{i}^{l}(1 \leq \forall l \leq W$, $\forall i \in V), 0 \leq S_{i, t}^{l} \leq 1$, which represents the degree of interest in the content of the article that $v_{i}^{l}$ intends to post, is randomly selected. 


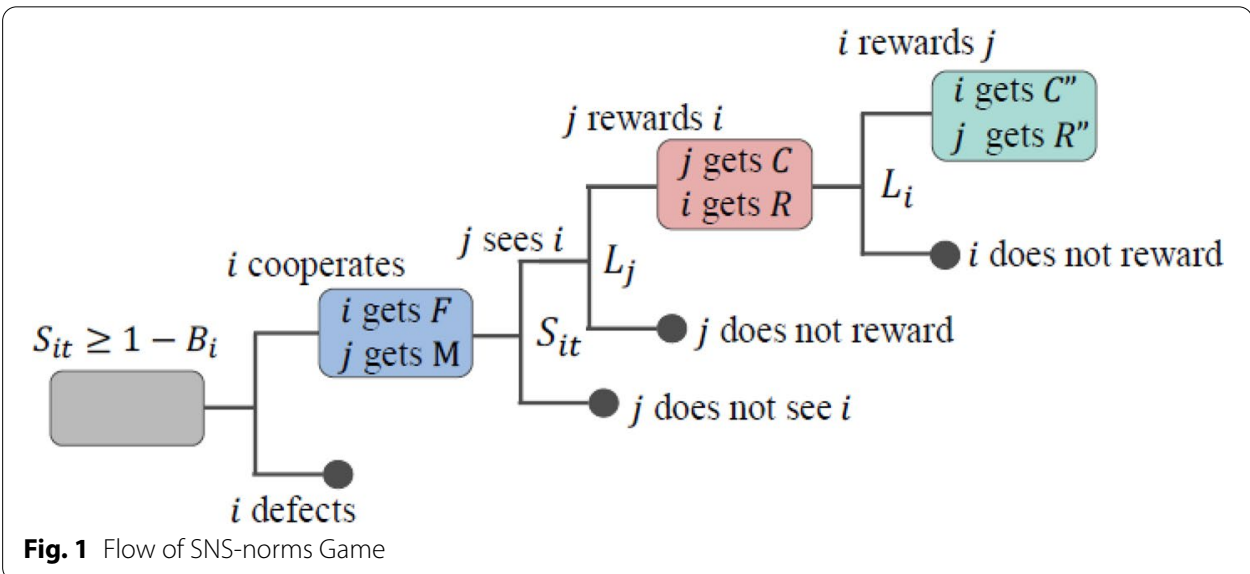

Fig. 1 Flow of SNS-norms Game

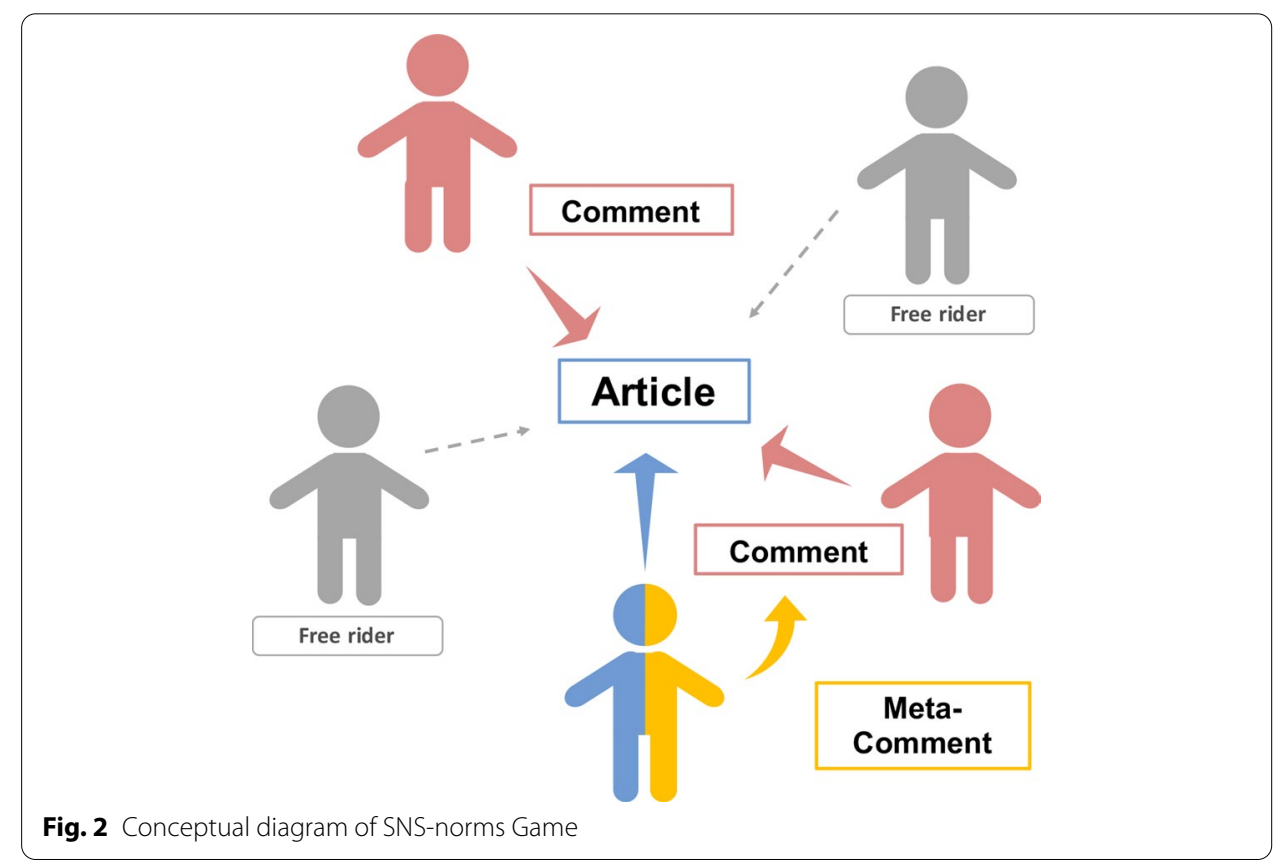

In the first step, agent $v_{i}^{l}$ in the $l$-th world posts an article and pays a cost $F(\leq 0)$ if $S_{i, t}^{l} \geq 1-B_{i}^{l}$. An article post is regarded as a contribution to the SNSs so the neighboring agent $v_{j}^{l} \in N_{i}^{l}=\left\{v_{j}^{l} \mid\left(v_{i}^{l}, v_{j}^{l}\right) \in E^{l}\right\}$ can receive benefit $M(>0)$ as a reward by reading the posted article. In the second step, $v_{j}^{l} \in N_{j}^{l}$ posts a comment on the posted article with probability $L_{j}^{l}$. Then, $v_{j}^{j}$ pays cost $C(\leq 0)$, while the article writer $v_{i}^{l}$ who posted the original article receives reward $R>0$. Posting a comment on posted article is regarded as giving a reward to the agent that posted the original article, which can be seen as another contribution behavior.

Then, in the third step, $v_{i}^{l}$ may provide a meta-comment to $v_{j}^{l}$, the agents that commented, with probability $L_{i}^{l}$. If $v_{i}^{l}$ posts a meta-comment, it pays cost $C^{\prime \prime}(<0)$, and $v_{j}^{j}$ receives meta-reward $R^{\prime \prime}(>0)$. These steps are done in a game turn of $v_{i}$, and all agents in $V^{l}$ (for $\left.1 \leq \forall l \leq W\right)$ complete the game turn in a round. 
Table 1 lists the cost and reward parameters used in this paper, where their values in this table were defined on the basis of the experiments done by Axelrod [7] and are also identical to those used in other studies $[3,9,15]$ so that we could examine the effect of the multiple-world GA properly. Table 2 lists the agent's total reward (or payoff) in a game turn, where $N_{c}(a)$ is the number of comments posted by neighboring agents on article $a$, and $N_{c c}(a)$ is the number of meta-comments posted in reply to the received comments. $N_{m}(c)$ is the number of meta-comments on comment $c$, which is 0 or 1 in the SNS-norms game. In this table, "cooperate" means "posting articles" and/or "making comments," and "defect" means "just reading articles/comments without posting or commenting." Note that we assume that the reward for posting an article follows the Weber-Fechner law [34] and increases logarithmically with the number of comments received [35].

The game runs in parallel on each duplicated network, and the evolution phase comes when all agents complete four rounds in a generation. The fitness value of the agent on each network is defined as the total rewards of the agent in a generation; thus, the siblings of an agent have different fitness values because their neighboring agents have different strategies.

\section{Evolution by multiple-world GA}

At the end of each generation, each agent in all duplicated networks simultaneously evolves its own strategy. The evolution of the multiple-world GA proceeds by the following three operations: selection, crossover, and mutation. The main feature of multipleworld GA is that agent $v_{i}^{l} \in \bigcup_{l=1}^{W-1} V^{l}$ selects itself as one parent and selects another parent from its siblings $A_{i}^{-l}=A_{i} \backslash\left\{v_{i}^{l}\right\}$ with the probability distribution $\left\{P_{i}^{l}\right\}_{i \in A_{i}^{-l}}$, where

$$
P_{i}^{l}=\frac{\left(f\left(v_{i}^{l}\right)-f_{\text {min }}\right)^{2}}{\sum_{v \in A_{i}^{-l}}\left(f(v)-f_{\text {min }}\right)^{2}},
$$

where $f\left(v_{i}^{l}\right)$ is the fitness function whose value is the fitness value of the given agent $v_{i}^{l}$ (see Table 2), and $f_{\text {min }}=\min _{v \in A_{i}} f(v)$. Note again that agents in $A_{i}$ stand in the same position in all duplicated networks. For $1 \leq \forall l \leq W-1, v_{i}^{l}$ s gene for the next generation is generated from the selected sibling and itself, and then the uniform crossover and flip-bit mutation with a probability of 0.005 for each bit are applied to $v_{i}^{l}$ s gene. Therefore, for example, if $W=10$, approximately one bit of the gene of a sibling of a master agent is inverted every four generations (because $9 \times 6 \times 0.005=0.27$ ). Then, the sibling agent with the generated gene is placed at $v_{i}^{l}$ for the next generation.

On the other hand, the gene of agent $v_{i}^{W} \in V^{W}$ in the next generation is set to that of the agent whose fitness value is highest in $A_{i}$. This operation indicates that the $\mathrm{W}$ th world consists of all agents whose fitness values are highest in $A_{i}=\left\{v_{i}^{1}, \ldots, v_{i}^{W}\right\}$ for $1 \leq \forall i \leq n$. Therefore, $W$-th world can be seen as a trial execution world because it consists of the agents who possibly have the best genes in the previous generation from the viewpoints of individual agents but may have interactions with different neighbors. Thus, we can assume that the agents in $V_{i}^{W}$ are provisional agents that have provisional genes, and these genes are expected to become the most suitable ones after a sufficient number of evolution processes. Note that the resulting gene of agent $v_{i} \in V$ in a certain 

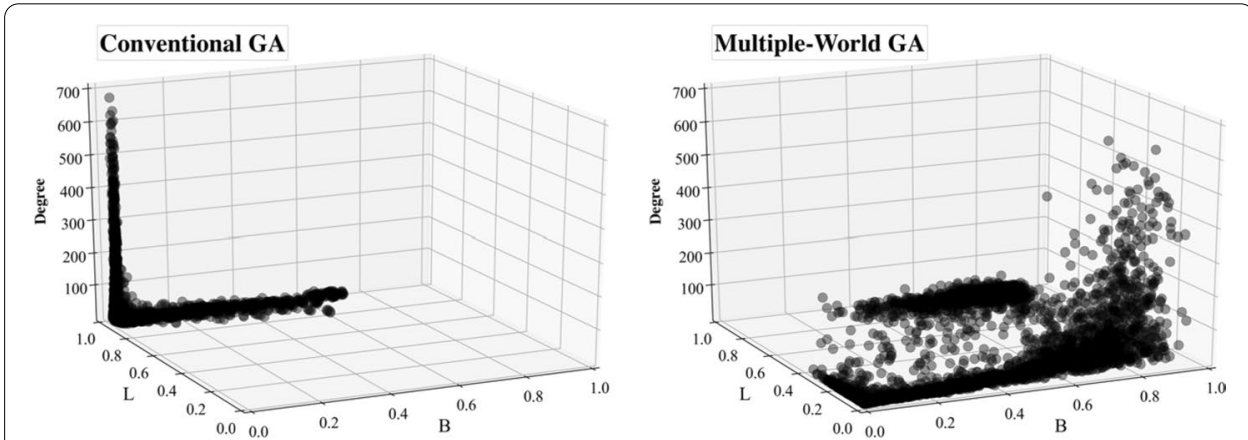

Fig. 3 Distribution of probability of posting rate $B_{i}$ and comment rate $L_{i}$ (connecting nearest-neighbor network)

generation is defined as the gene of $v_{i}^{W} \in A_{i}^{W}$. This is used to generate the dynamic process of gene evolution.

\section{Experiments and discussion}

\section{Experimental setting}

We conducted the experiments of SNS-norms games running on a network generated by the actual Facebook network [33] and the ten associated ego networks (these networks are simply called the Facebook network and Facebook ego networks after this) and investigated how agents individually learn (co-evolve) their suitable strategies, which are specified by the probability of posting article $B$ and the probability of posting comment $L$ through interactions with their neighboring agents. The network characteristics of the Facebook network we used are listed in Table 3. We conducted these experiments using the multiple-world GA for 10,000 generations, and our results were compared with those using the conventional GA [3,9] in which agents probabilistically copy the better strategies from neighboring agents for the next generation. The number of world $W$ in the multiple-world GA is 10, and the mutation rate of genetic operation in both multipleworld GA and conventional GA is 0.005 . We also conducted experiments using artificial complex networks, connecting nearest-neighbor networks, to compare their distributions of strategies in the artificial networks and the Facebook network. These connecting nearest-neighbor networks were generated with the conversion probability $u=0.955$ which represents the probability that one potential link is converted into an actual link and was defined so that the average degree of the connecting nearest-neighbor networks is almost identical to that of the Facebook network. Other network characteristics of the connecting nearest-neighbor networks are also shown in Table 3. The values of $B_{i}$ and $L_{i}$ plotted in the figures below are those of the provisional agents in $A_{i}^{W}$ in the specified generations. The results shown below are the average values of independent 100 experimental runs based on different random seeds, unless otherwise noted. Therefore the values of $B_{i}$ and $L_{i}$ plotted in the figures take continuous values.

\section{Comparison between conventional GA and multiple-world GA: strategy distribution}

First, let us compare the distributions of strategies that agents took in the connecting nearest-neighbor networks and the Facebook network by using the conventional 

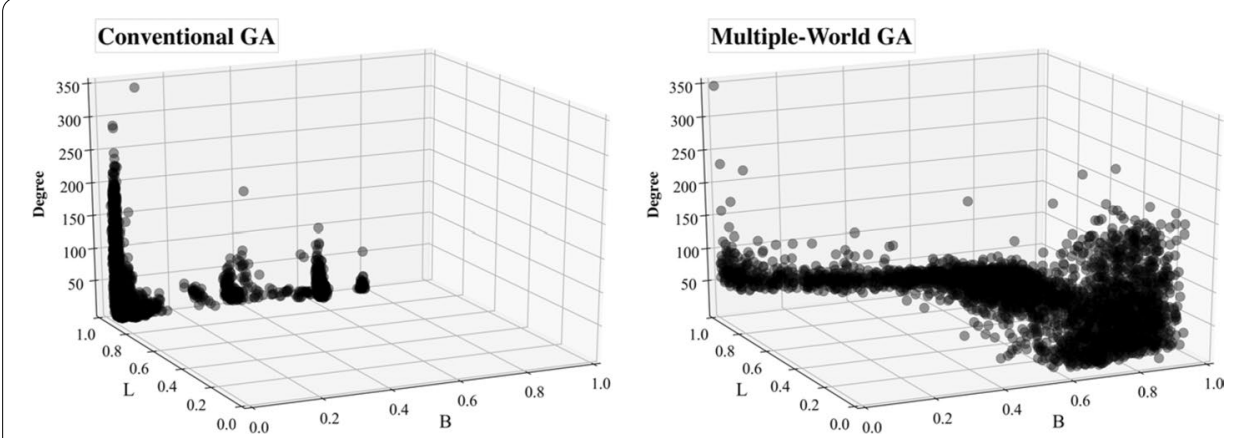

Fig. 4 Distribution of probability of posting rate $B_{i}$ and comment rate $L_{i}$ (Facebook network)

GA and the multiple-world GA. The experimental results are shown as scatter graphs (Figs. 3 and 4).

Let us look at the scatter graphs of the connecting nearest-neighbor networks when using the conventional GA (Fig. 3). We can see that all agents have similar strategies in which $L$ is near 1.0, and many agents have low values of $B$, regardless of their degree numbers, because in the evolution process, all agents were likely to take on the better strategies of their neighboring agents. In particular, the hub agents, which have much higher degree numbers than other agents, were likely to earn more rewards; its neighboring (and also their neighboring) agents tended to copy the strategies of nearby hub agents. However, all agents have high $L$ values; we think that such uniform strategies are not realistic in the actual SNSs.

On the other hand, Fig. 3 also indicates that when using the multiple-world GA, the agents' evolved strategies were diverse depending on their network locations and the strategies taken by their neighboring agents. Here, we classified agents into four groups: free riders (that have low values of $B_{i}$ and $L_{i}$ ), comment writers (that have a low value $B_{i}$ and a high value of $L_{i}$ ), article writers (that have a high value of $B_{i}$ and a low value of $L_{i}$ ), and active agents (that have high values of $B_{i}$ and $L_{i}$, so they frequently post articles and comments). From Fig. 3 , it can be seen that some free riders appear in the connecting nearest-neighbor networks because just reading articles posted by their neighboring agents is often beneficial, and this is quite common in an actual SNS (and in human society). This suggested that the results using the multipleworld GA reflected users' activities in the real world. These results are consistent with our previous results [15].

In the Facebook network, agents took more varied strategies, as shown in Fig. 4. When using the conventional GA, we can observe some clusters of evolved strategies. This is because the actual network consists of a number of clusters (communities) that have different network characteristics, and agents in each community have similar strategies, i.e., similar values of $B_{i}$ and $L_{i}$. In contrast, agents in the Facebook network have diverse strategies. One interesting property observed in Fig. 4 is that, unlike the results of the connecting nearest-neighbor networks, no free riders appeared in the Facebook network; none of the agents think that free riding is a good strategy. Another property observed in the Facebook network is that there are many comment writers. It seems costly to keep making many comments, and so there were 

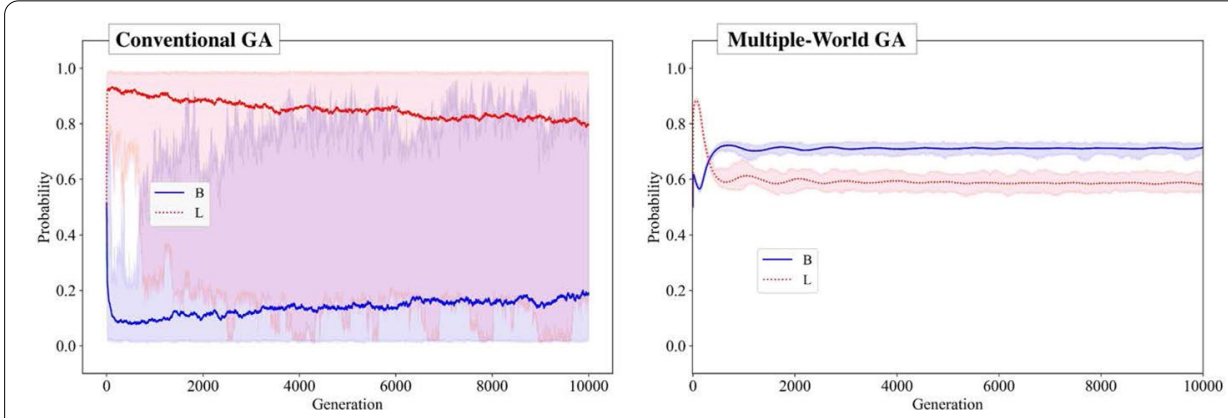

Fig. 5 Probability of average posting rate $B$ and average comment rate $L$
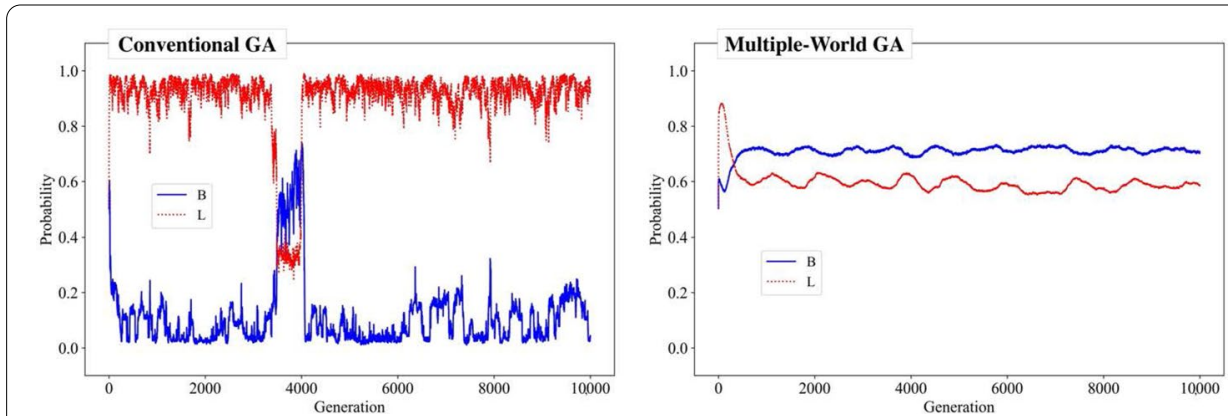

Fig. 6 Probability of average posting rate $B$ and average comment rate $L$ in a single run

no comment writers in the connecting nearest-neighbor networks. The conditions under which comment writers can have benefits is when their neighbors also have high $L$ (and moderate $B$ ) and are connected densely; otherwise, free riding is more beneficial. These properties can be attributed to only the characteristics of the network, and we think that it is necessary to investigate these characteristics.

\section{Comparison between conventional GA and multiple-world GA: Average posting/comment} rates, fitness values, and dispersion

We would like to confirm whether the agents could identify better strategies and so gain higher fitness values using the multiple-world GA than those obtained by using the conventional GA, which is often used in the existing research.

Figure 5 shows the transition of the average of agents' probabilities of posting article $B_{i}$ and comment $L_{i}$ in the SNS-norms game on the Facebook network(the indices of agent $v_{i}$ are omitted in this and other figures) when using the multiple-world GA and the conventional GA. Figure 7 shows the transition of average fitness values of all agents. Note that in these figures, the bold lines indicate the average values of the 100 times runs, and the light colored vertical lines indicate the max and min values. We can see in Fig. 5 that $B$ and $L$ converged to around 0.7 and 0.6 , respectively, when using the multiple-world GA, while they were 0.2 and 0.8 , respectively, at the end of generation when using the conventional GA. Furthermore, the values of dispersion, which is defined as the difference between the max and min values, were quite large when using the GA; this indicates that the agents' strategies were extremely unstable. On the other hand, the values 
of dispersion when using the multiple-world GA were quite small, so their strategies seemed much stabler.

We can see the reason for this stability and instability from Fig. 6, which indicates the result of a certain single experimental run and how agents identify their strategies in detail. The graph of the conventional GA in Fig. 6 shows that the average posting article rate $B$ and comment rate $L$ of all agents fluctuated ( $B$ is mainly between 0 and 0.2 , and $L$ is mainly between 0.82 and 1.0). Furthermore, $B$ and $L$ suddenly but temporarily changed in around the 3600th to 4000th generations in Fig. 6; this is probably the result of mutation, and such changes drastically affected the entire strategies. In contrast, the average $B$ and $L$ when using the multiple-world GA in Fig. 6 were stabler and were not significantly different from those in Fig. 5. Of course, the mutation occurred, but such changes were negated by the surrounding agents. Note that we can see the strategies fluctuated rapidly in the earlier generations ( $L$ spiked temporarily, and $B$ dropped temporarily). We found that these sudden changes were the result of searching for the better strategies, which were also affected by the neighboring agents that also searched for their better strategies.

Finally, we insist that the strategies identified by the multiple-world GA were better than those identified by the conventional GA. As shown in Fig. 7, the average fitness values converged to approximately 50 at the end of generation when using the conventional GA but converged to over 200 and were stable when using the multiple-world GA.

\section{Strategy distribution on the whole Facebook network}

To understand the effectiveness of the multiple-world GA for agents to identify their own appropriate strategies based on the network locations and the surroundings, we investigated the strategies that agents on the Facebook network took during the evolution. First, we show the distribution map of strategies on the example network, which is identical to that of Fig. 6, at the end of evolution in Figs. 8 and 9 when using the conventional GA and the multiple-world GA, respectively. In this figure, nodes are agents on the network, and the edges correspond to the connections among agents. The circle sizes of the nodes represent the probability of posting articles (a large node has a high $B$ ), and the color of the nodes represents the probability of commenting (as $L$ increases, the color turns from blue to red).

As shown in the strategy distribution map when using the conventional GA (Fig. 8), almost all agents learned to have a low posting article rate $B$ and high comment rate $L$, and these values were quite similar to those of their neighboring agents. Although only the community at the bottom (indicated by an arrow in Fig. 8) has comment rates that are $L$ different from other agents, the agents in other communities had similar strategies. When using the multiple-world GA, in contrast, we can observe all clusters (communities in the Facebook network) have both red and blue nodes, and a number of communities contain a few subcommunities whose members have different strategies (mainly blue nodes) than those of other agents, as shown in the strategy distribution map in Fig. 9.

\section{Dynamics of Strategies in the Facebook network}

Next, we analyzed the dynamics of the strategies selected by agents in the Facebook network. Because Figs. 5 and 7 suggest that agents' strategies changed by the first 
Table 1 Parameter values of costs and rewards in experiments

\begin{tabular}{llr}
\hline Parameter & Description & Value \\
\hline$F$ & Cost of posting article & -3.0 \\
$M$ & Reward for reading article & 1.0 \\
$C$ and $C^{\prime \prime}$ & Costs of comment and meta-comment & -2.0 \\
$R$ and $R^{\prime \prime}$ & Rewards for receiving comment and meta-comment & 9.0 \\
\hline
\end{tabular}

Table 2 Sum of rewards (costs) for each game turn

\begin{tabular}{lll}
\hline Type of action & Cooperate & Defect \\
\hline Article (post article $a)$ & $F+R \times \log _{e}\left(N_{c}(a)+1\right)+C^{\prime \prime} \times N_{c c}(a)$ & 0 \\
Comment (post comment $c)$ & $M+C+R^{\prime \prime} \times \log _{e}\left(N_{m}(c)+1\right)$ & $M$ \\
\hline
\end{tabular}

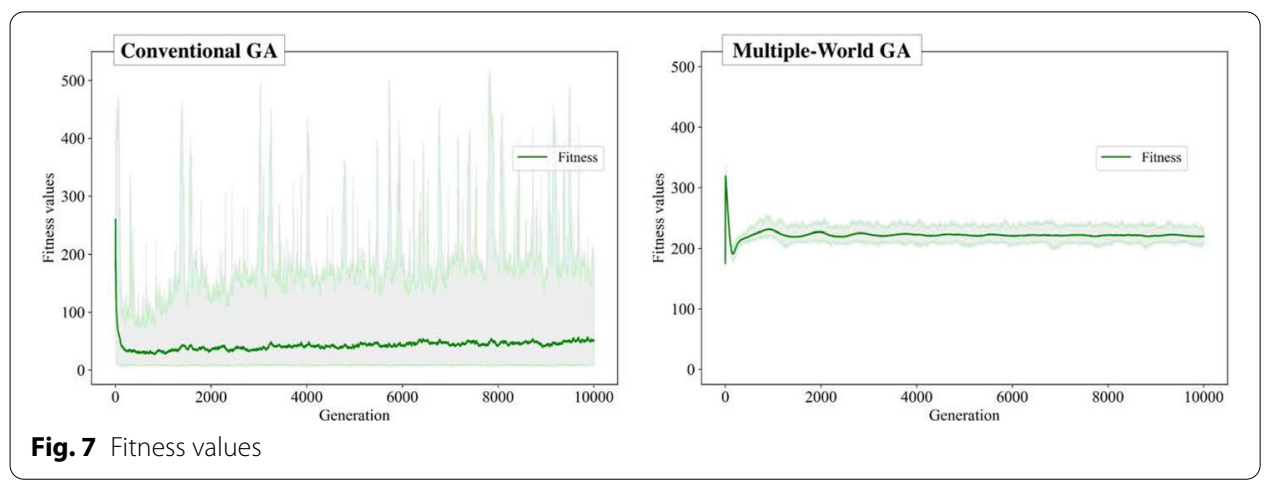

400 generations and then stabilized, we plotted the strategies evolved in agents every 100 seconds from the 0th generation in Fig. 10 and 11. The agents in Fig. 10 have low degree (less than 100), and the agents in Fig. 11 have high degree (more than 100).

Figures 10a and 11a indicate that most of high degree agents were comment writers who have high $L$ and low $B$ values, and most of low degree agents were active agents who have high $B$ and high $L$ in earlier generations. The motivation for the comment writers is to receive comments on their comments (meta-rewards) from the active users in these generations, and actually, active agents gained many comments from comment writers by posting articles. However, once the active agents realized that they could gain more rewards by posting articles without commenting to anyone, some active users with low degrees gradually changed to be article writers, since they did not need to pay costs for posting comments (Fig. 10b, c). Hence, because the comment writers could not receive sufficient rewards, the average fitness value rapidly decreased as shown in Fig. 7, and thus, most of comment writers changed to be article writers to give up receiving rewards by commenting (Fig. 11bd); during this process, the average fitness value gradually increased because they still could gain comments from remaining active agents in Fig. 10d. 


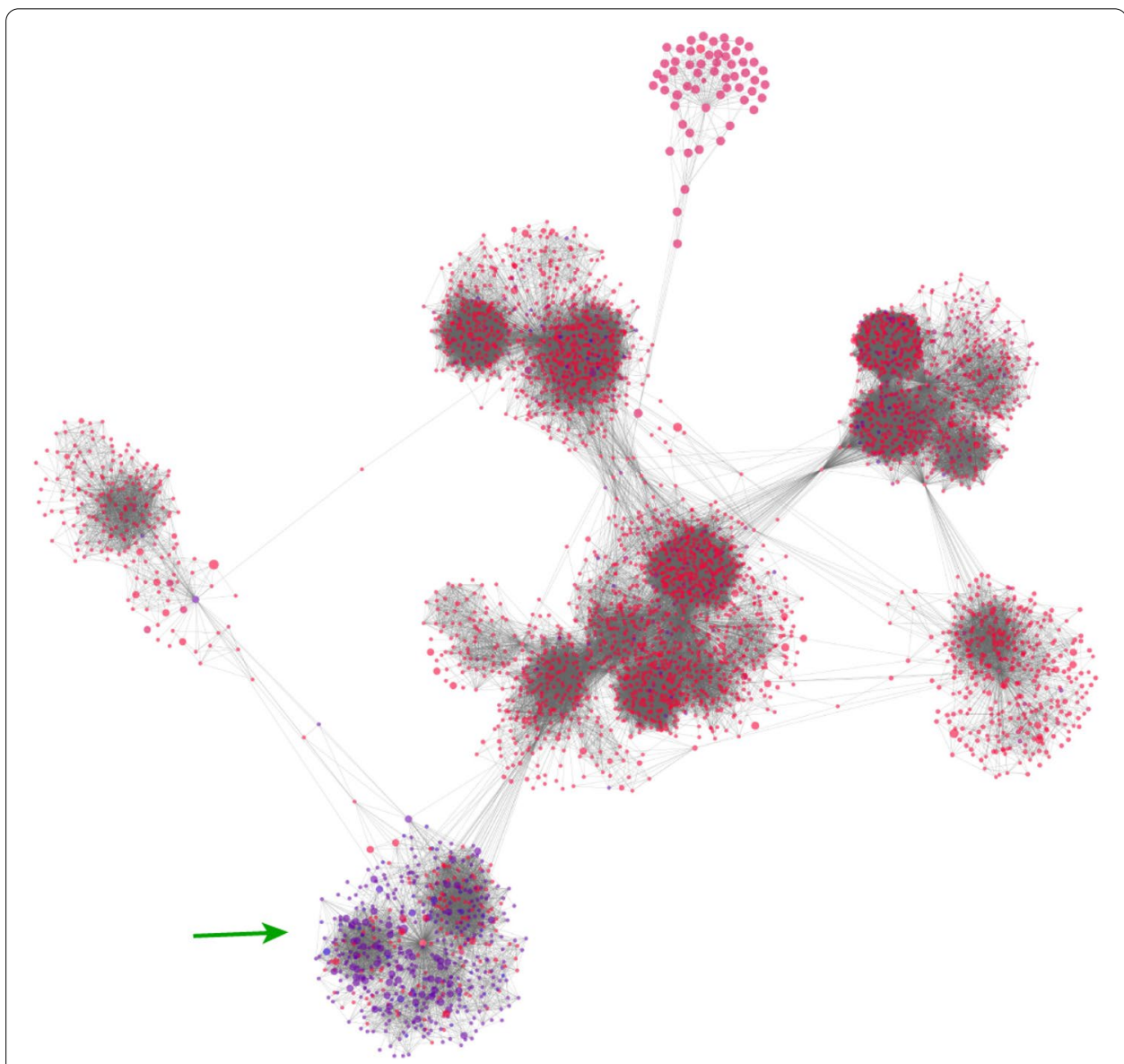

Fig. 8 Strategy distribution on Facebook network (Conventional GA). The circle sizes of the nodes represent the probability of posting articles (a large node has a high B), and the color of the nodes represents the probability of commenting (as $L$ increases, the color turns from blue to red)

\section{Influence of rewards for comment and meta-comment}

We examined how the ratio of the rewards for comment to the cost for comment and meta-comments in various networks affect their strategies and what types of networks are sensitive to this ratio in the second experiment because the value of $L$ seems to affect agents' strategies from the previous results. For this purpose, we extracted 10 ego networks from the Facebook network used in the previous experiment; the network characteristics of these ego networks are listed in Table 4, where their network ID numbers are assigned in ascending order of the average degrees. We ran the 10 experimental trials using the multiple-world GA with different rewards of comment and meta-comment, $1 \leq R=R^{\prime \prime} \leq 20$, for each ego network until the 10,000th generation. The other parameter values were set to the same values as those of the previous experiments (Table 1). The results we show below are the average of 10 experimental trials for each ego network with the 10 random seeds.

Figures 12 and 13 show the average posting article rate $B$ and comment rate $L$ between the 9000th and 10,000th generations for some rewards for comment $R$. Figure 12 indicates that the posting article rate $B$ gradually increased with increasing reward for comment until $R=6$ in all ego networks. Then, it suddenly rose in all networks, especially 


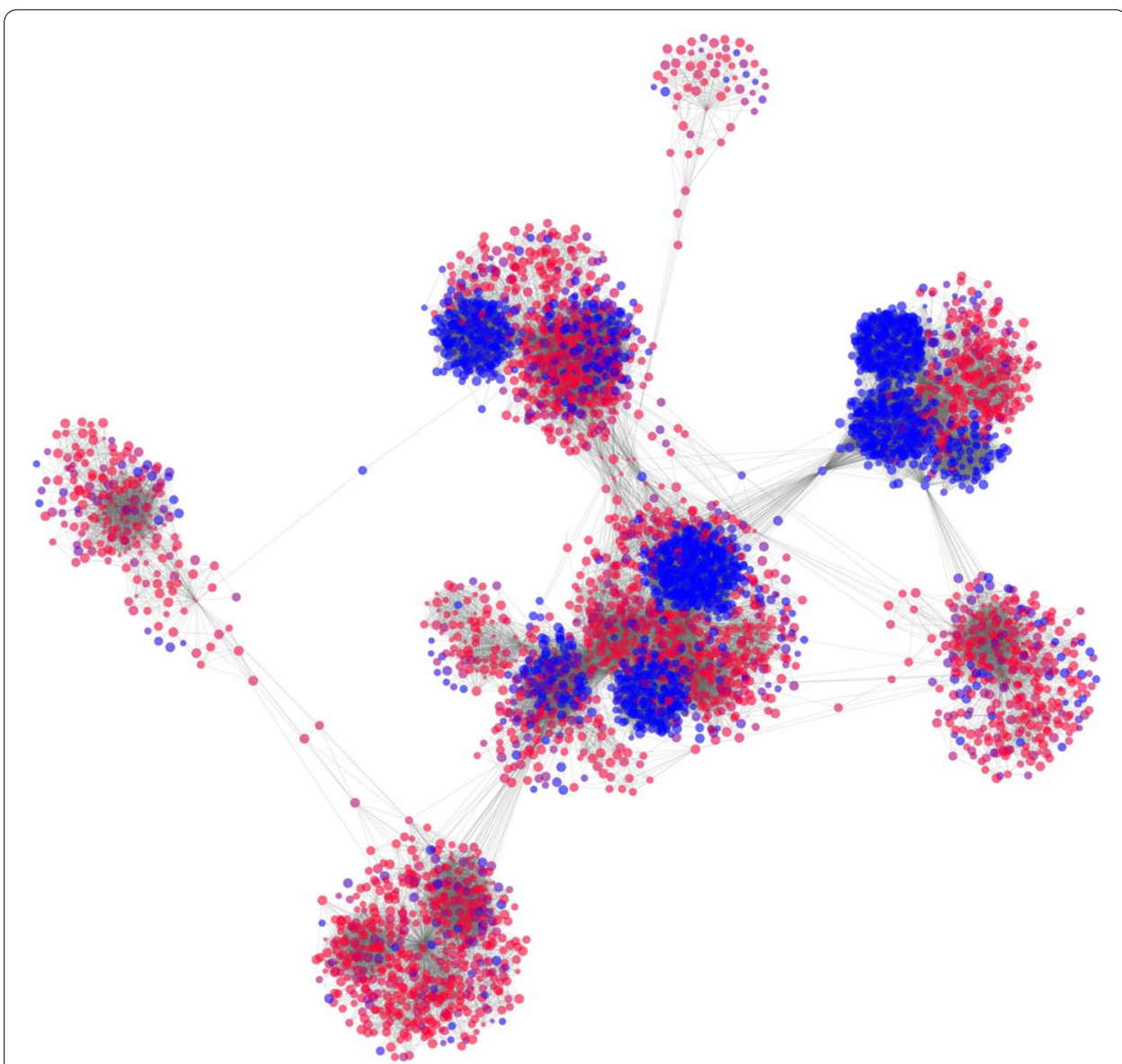

Fig. 9 Strategy distribution on Facebook network (multiple-world GA). The circle sizes of the nodes represent the probability of posting articles (a large node has a high B), and the color of the nodes represents the probability of commenting (as $L$ increases, the color turns from blue to red)

in a few networks with low degrees whose network ID numbers are 1 and 2 (see Fig. 12), and it gradually increased in accordance with the values of $R$ when $R \geq 7$. We can also find that $L$ decreased with increasing of $R$ until $R=6$ (from Fig. 13) in all ego networks (this is the opposite change of $B$ in Fig. 12). Then, $L$ also soared (the higher the average degrees, the more slowly they rose). However, when $R \geq 9, B$ temporarily decreased in networks whose ID numbers are 7, 8, 9, and 10 in Fig. 13). From these results of the second experiment, the average values of $B$ and $L$ of all agents in the networks whose average degrees are smaller are likely to be influenced by the value of $R$.

Let us look at Fig. 13 between $R\left(=R^{\prime \prime}\right)=1$ and 6; the increase of the comment reward $R$ can be seen as an increase of the incentive to comment on posted articles, but the value of $L$ decreased, while the value of $B$ increased. This is because if $R^{\prime \prime}$ gets higher and $L$ is high, an agent that posts an article is likely to received many comments and the agent has to reply to these many comments; this is costly. Thus, a lower value of $L$ became the dominant strategy.

In the same way, when $R \geq 7$ agents in the networks whose average degrees were higher receive many comments from their neighbors, they have to reply to these comments. Thus, their increases of $L$ became were moderate. Furthermore, $B$ of agents in the 

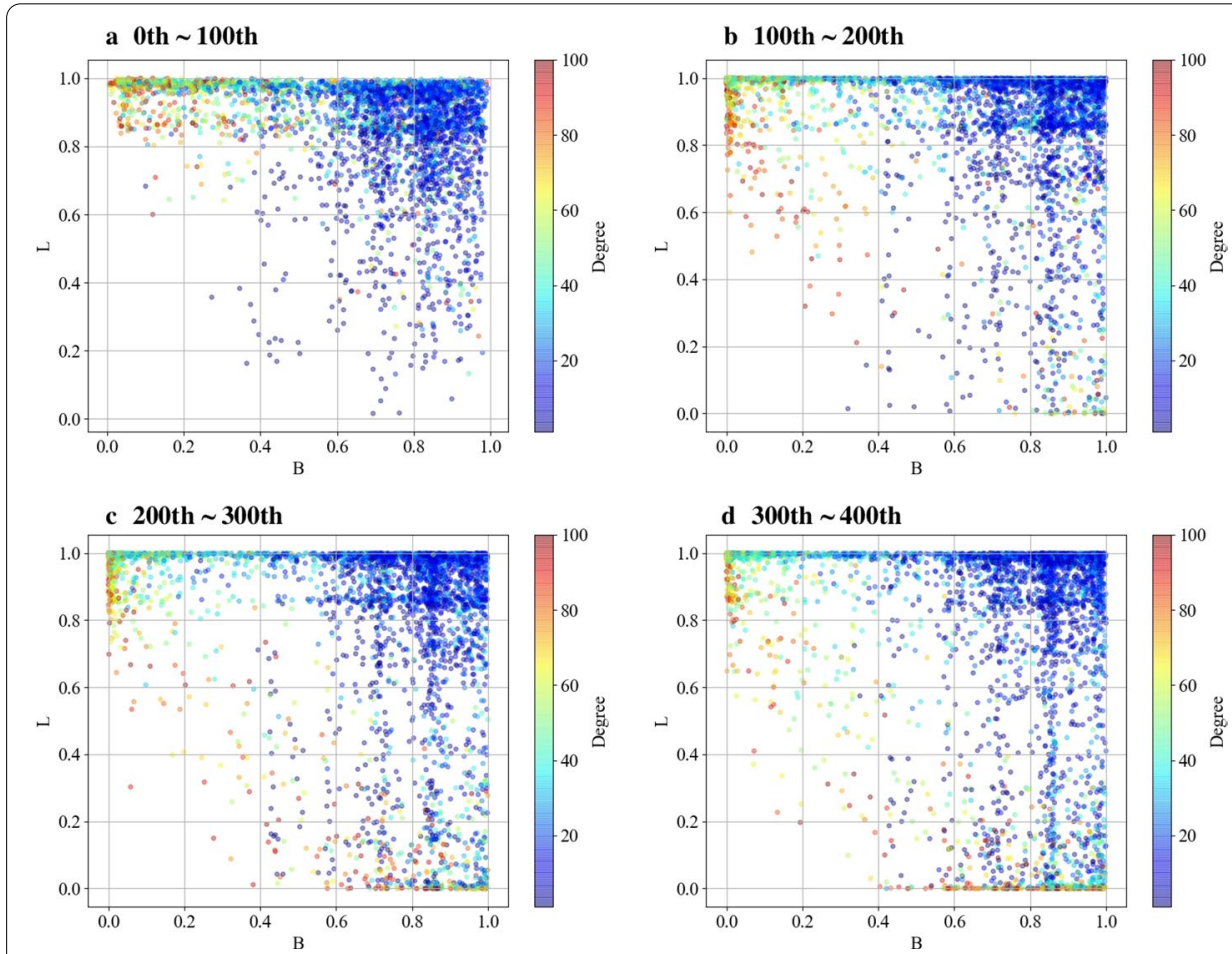

Fig. 10 Dynamics in co-evolved strategies (probability of average posting rate $B$ and average comment rate L) on Facebook network (low degree agents)

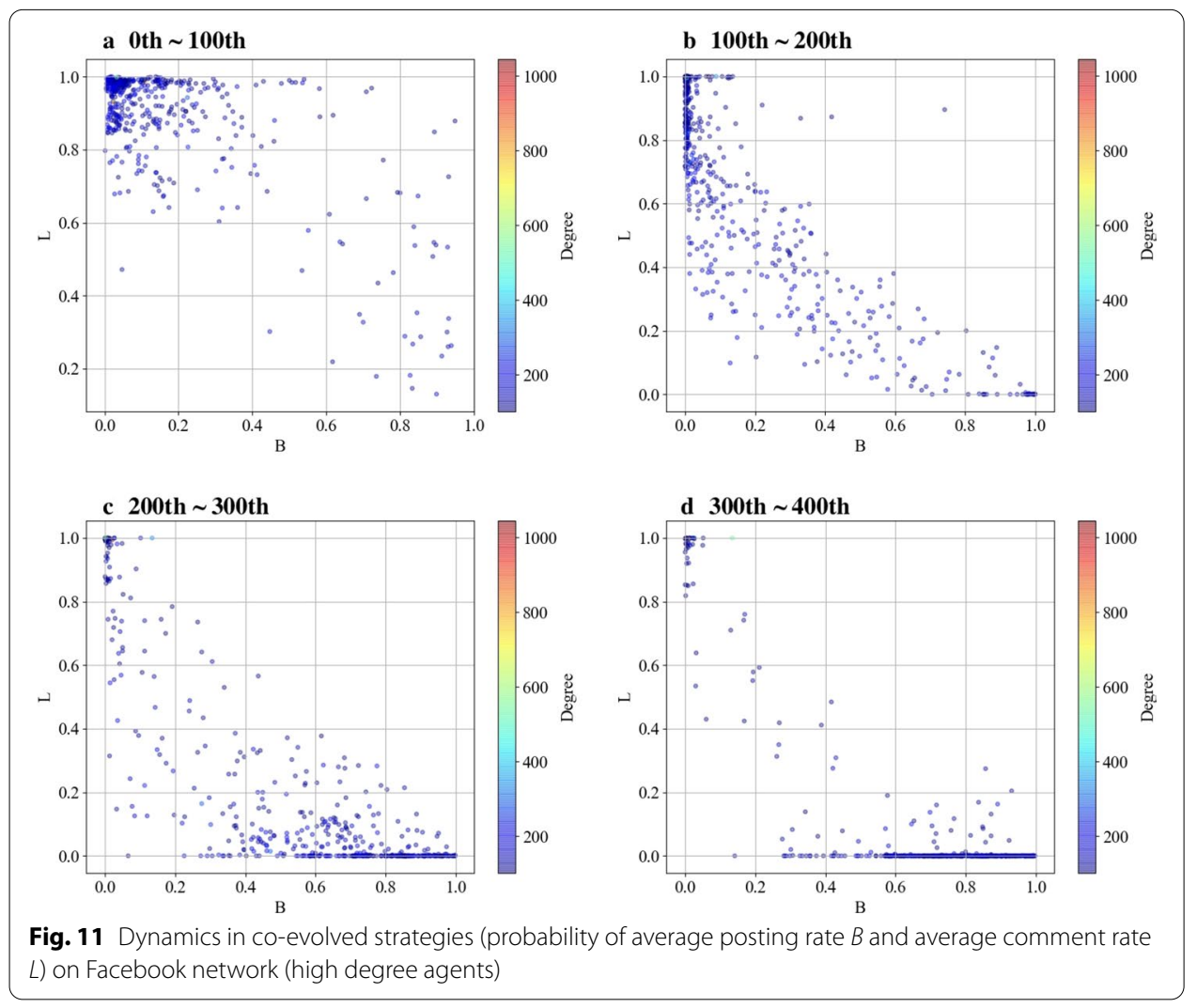




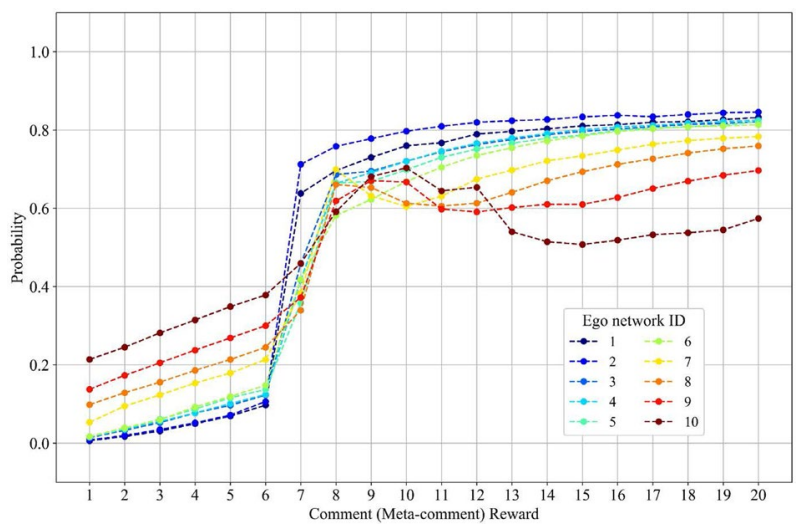

Fig. 12 Relationship between posting article rate $B$ and the value of rewards $R\left(=R^{\prime \prime}\right)$

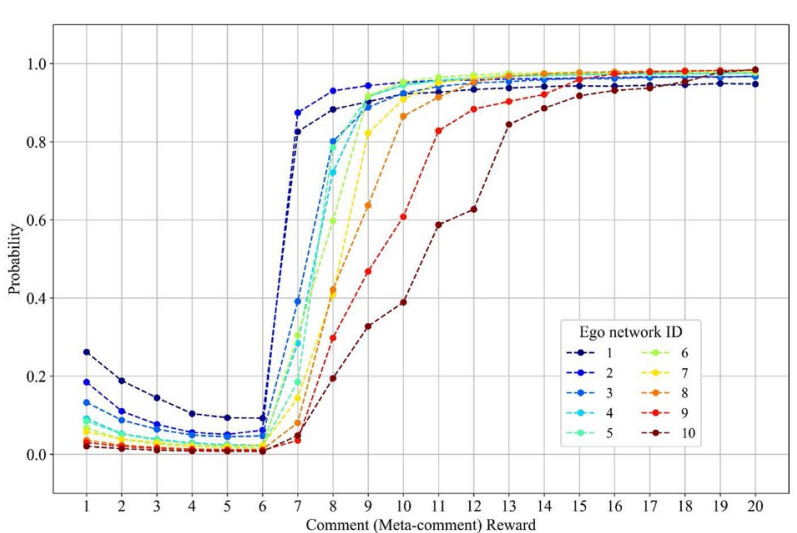

Fig. 13 Relationship between comment rate $L$ and the value of rewards $R\left(=R^{\prime \prime}\right)$

Table 3 Parameters and characteristics of the Facebook and connecting nearest-neighbor networks

\begin{tabular}{llll}
\hline Parameter & Description & Facebook & $\begin{array}{l}\text { Connecting } \\
\text { nearest- } \\
\text { neighbor }\end{array}$ \\
\hline$n$ & Number of agents & 4039 & 4039 \\
$\langle k\rangle$ & Average degree (average number of friends) & 43.691 & 43.483 \\
$C C$ & Average clustering coefficient & 0.606 & 0.449 \\
$A L$ & Average characteristic path length & 3.693 & 3.566 \\
$R$-squared & R-squared for fitted power-law line & 0.809 & 0.867 \\
\hline
\end{tabular}

high-degree networks temporarily decreased to avoid the cost rise caused by many comment replies when $R \geq 9$. Therefore, we can say that the increase of $R$ led to more incentive to post articles but this was outweighed by the cost of replying to many comments from many neighboring agents. We think that this phenomenon is social network fatigue, which has been discussed by Yamakami et al. [23]; they revealed in their empirical study that getting tired of commenting on friends' comments on an SNS was a high-ranking 
Table 4 Parameters and characteristics of the Facebook ego networks

\begin{tabular}{llllll}
\hline $\mathrm{ID}$ & $\langle k\rangle$ & $n$ & $C C$ & $A L$ & $R$-squared \\
\hline 1 & 5.6 & 44 & 0.455 & 2.57 & 0.395 \\
2 & 8.8 & 40 & 0.725 & 1.95 & 0.146 \\
3 & 15.13 & 324 & 0.522 & 3.75 & 0.804 \\
4 & 18.03 & 532 & 0.546 & 3.45 & 0.634 \\
5 & 19.7 & 168 & 0.534 & 2.43 & 0.503 \\
6 & 22.6 & 148 & 0.679 & 2.69 & 0.0469 \\
7 & 28.5 & 224 & 0.544 & 2.52 & 0.431 \\
8 & 35.68 & 775 & 0.471 & 3.04 & 0.557 \\
9 & 51.74 & 1034 & 0.526 & 2.95 & 0.683 \\
10 & 80.34 & 744 & 0.638 & 2.56 & 0.343 \\
\hline
\end{tabular}

reason for quitting the SNS. Finally, note that we conducted the same experiments using the conventional GA and found that a similar phenomenon occurred, but the agents' strategies fluctuated and were unstable.

\section{Discussion}

We summarize a number of characteristics of the Facebook network using the multipleworld GA found in our experiments by comparing them with those of the connecting nearest-neighbor networks that were also shown in our conference paper [15]. First, we can see the experimental results using the multiple-world GA were more realistic than those using the conventional GA, and all agents could evolve to have diverse strategies depending on the locations and the strategies of surrounding agents. Furthermore, almost all agents had higher fitness values; this was consistent with the results reported in Miura et al. 's study [15]. One interesting and unexpected characteristic found in the Facebook network is that no agents evolved to behave as free riders. Because agents can get benefits by just reading articles and comments with no cost, free riding seems a good strategy; actually, this is an equilibrium in a kind of dilemma situation. Because we only focus on the network structure, our experimental results suggest that some sort of cooperation can be realized by carefully designing the connections between agents.

We suppose that being connected with friends who have similar network characteristics in the Facebook network could maintain the cooperative behavior. As a common example, the user builds dense friendship network on the Facebook like in the real world and actively interacts with their friends even though the user's friends are not so many on the Facebook. Considering the network characteristics of connecting nearest-neighbor model, some agents sparsely connected to neighbors whose network features are not similar to them at all because of the algorithm of generating networks. Under the situation that the agents are strongly affected their neighboring behaviors, users' cooperative strategies in a sparse network easily collapse. Degree of connectivity between friends with similar characteristics is partly measured by assortativity [36-38], but there are a number of different definitions of assortativity; we will investigate the relationship between such various assortativity measures and evolution of cooperation in the future. We would like to note that this discussion is based on our recent results. 
Second, from the experiment that investigates the effect of the relative relationship between variable rewards and costs on the evolved strategies in the second experiment, we found that agents in the ego networks, whose average degree is low, are likely to be sensitive to the increase of reward $R\left(=R^{\prime \prime}\right)$, which is given to the agents when they receive comments and meta-comments. However, the agents with high degrees did not simply increase the comment rate $L$ as $R$ increased. On the contrary, $L$ temporarily decreased because the cost of replying to many comments of its neighboring agents was very large, so the increase of the average of $L$ was slow, as shown in Fig. 13, and the average value of $B$ also temporarily decreased to reduce the number of comments and meta-comments in the ego networks with high average degrees, as shown in Fig. 12. In general, it is costly to keep the value of $L$ high; therefore, agents could not take the strategy of comment writers in the connecting nearest-neighbor network, but some agents in Facebook took it (but not that many; we think that such comment writers exist in actual SNSs). This characteristics was also caused by the structure of the network.

Finally, the previous discussion also explains the strategy distribution shown in Fig. 9. Almost all agents in the Facebook network were expressed by the red and relatively large circle nodes, meaning that they were active in posting articles and commenting. However, we can also see a number of clusters of blue nodes in a large community, meaning the strategy of the article writer that rarely comments on other articles. We think that agents in these clusters were densely connected and so can maintain the strategy of article writers with each other. At first, agents in this type of subcommunities took the strategy of comment writers, like other agents. Then, due to the mutation, a few agents posted articles, and because their neighbors are mostly comment writers and all agents were connected densely, they received high rewards and kept the strategy. Then, they could find that even if they did not comment, they could receive sufficient rewards with less cost, so they became article writers (which are shown as blue circles). We think that a similar phenomenon has also been reported in high-clustered scale-free networks [39] and we would like to investigate the relationships with these studies.

\section{Conclusion}

We simulated the game-theoretic model of social networking services (SNSs) on the real Facebook network with the multiple-world genetic algorithm (multiple-world GA), a coevolutionary algorithm, to understand the users' diverse strategies and the process of evolution on the SNS and compared the evolved strategies with those in the artificial networks, the connecting nearest-neighbor networks. Through these comparisons, we found that agents in the Facebook network did not take the strategy of free riding, which is often an equilibrium of public-goods games like the SNS-norms game, although many free riders appeared in the connecting nearest-neighbor networks. Agents in the Facebook network also did select the strategies of the comment writers, who often comment on posted articles and comments by other agents but rarely post articles, but comment writers are usually costly and it is difficult for them to continuously receive sufficient rewards, so no comment writers were evolved in the connecting nearest-neighbor networks. These characteristics found in our experiments are caused only by some structural characteristics of the Facebook network, and to identify the reason is crucial future issue. 
We also compared the results with those of the conventional GA that is used in the existing studies with those of the multiple-world GA. Then, we confirmed that agents with the multiple-world GA evolved the diverse strategies that were suitable for their surroundings because they had higher fitness values. Thus, we believe that a plausible model of dynamic behaviors could be reproduced with the multiple-world GA to explain well the process of behavior selections in actual SNSs.

We plan to conduct a simulation of the co-evolutionary SNS model with the heterogeneous agents that have the individually different benefit/cost for the behaviors on SNSs; this study might be helpful to understand the users' cooperation behavior on the SNS in detail.

\section{Abbreviations}

SNSs: Social Networking Services; GAs: Genetic Algorithms; NPD: N-players Prisoner's Dilemma; SPs: Subprocesses.

\section{Acknowledgements}

Not applicable.

\section{Authors' contributions}

All authors participated in the discussion of designing model and planning experiments, and thus almost equally contributed to the work. YM FT and TS conceived the idea and YM designed and implemented the code for experiments. TS and YM composed the draft of the manuscript. All authors read and approved the final manuscript.

Funding

This work was in part supported by KAKENHI (17KT0044,19H02376,18H03498).

Availability of data and materials

The dataset used in this study is available from http://snap.stanford.edu/data.

Declarations

Competing interests

The authors declare that they have no competing interests.

\section{Author details}

${ }^{1}$ Department of Computer Science and Communications Engineering, Waseda University, 3-4-1 Okubo, Shinjuku, Tokyo 169-8555, Japan. ${ }^{2}$ Graduate School of Engineering, The University of Tokyo, 7-3-1 Hongo, Bunkyo, Tokyo 113-8656, Japan.

Received: 16 March 2020 Accepted: 20 February 2021

Published online: 10 March 2021

\section{References}

1. Faraj S, Johnson SL. Network exchange patterns in online communities. Org Sci. 2011;22(6):1464-80.

2. Lin K-Y, Lu H-P. Why people use social networking sites: an empirical study integrating network externalities and motivation theory. Comput Human Behav. 2011;27(3):1152-61.

3. Toriumi, F., Yamamoto, H., Okada, I.: Why do people use social media? agent-based simulation and population dynamics analysis of the evolution of cooperation in social media. In: Proceedings of the The 2012 IEEE/WIC/ACM International Joint Conferences on Web Intelligence and Intelligent Agent Technology-Volume 02, pp. 43-50 (2012). IEEE Computer Society.

4. Karamon J, Matsuo Y, Ishizuka M. Generating useful network-based features for analyzing social networks. Proc AAAI. 2008:2008:1162-8.

5. Saito, K., Masuda, N.: Two types of twitter users with equally many followers. In: 2013 IEEE/ACM International Conference on Advances in Social Networks Analysis and Mining (ASONAM 2013), pp. 1425-1426 (2013). https://doi. org/10.1145/2492517.2492575.

6. Qasem Z, Jansen M, Hecking T, Hoppe HU. Using attractiveness model for actors ranking in social media networks. Comput Soc Netw. 2017;4(1):3.

7. Axelrod R. An evolutionary approach to norms. Am Polit Sci Rev. 1986;80(4):1095-111.

8. Hirahara, Y., Toriumi, F., Sugawara, T.: Evolution of cooperation in sns-norms game on complex networks and real social networks. In: International Conference on Social Informatics, pp. 112-120 (2014). Springer.

9. Hirahara Y, Toriumi F, Sugawara T. Cooperation-dominant situations in sns-norms game on complex and facebook networks. New Gener Comput. 2016;34:273-90. https://doi.org/10.1007/s00354-016-0305-z.

10. Miura, Y., Toriumi, F., Sugawara, T.: Multiple world genetic algorithm to analyze individually advantageous behaviors in complex networks. In: Proceedings of the Genetic and Evolutionary Computation Conference Companion. GECCO '19, pp. 297-298. ACM, New York, NY, USA (2019). https://doi.org/10.1145/3319619.3321989. 
11. Miura, Y., Toriumi, F., Sugawara, T.: Multiple-world genetic algorithm to identify locally reasonable behaviors in complex social networks. In: 2019 IEEE International Conference on Systems, Man and Cybernetics (SMC), pp. 3665-3672 (2019). https://doi.org/10.1109/SMC.2019.8914277

12. Ebel H, Bornholdt S. Coevolutionary games on networks. Phys Rev E. 2002;66(5):056118.

13. Gupta D, Ghafir S. An overview of methods maintaining diversity in genetic algorithms. Int J Emerg Technol Adv Eng. 2012;2(5):56-60.

14. Shimodaira, H.: A diversity-control-oriented genetic algorithm (dcga): Performance in function optimization. In: Proceedings of the 2001 Congress on Evolutionary Computation (IEEE Cat. No. 01TH8546), vol. 1, pp. 44-51 (2001). IEEE

15. Miura, Y., Toriumi, F., Sugawara, T: Analysis of diversity and dynamics in co-evolution of cooperation in social networking services. In: International Conference on Complex Networks and Their Applications, pp. 495-506 (2019). Springer

16. Vázquez A. Growing network with local rules: preferential attachment, clustering hierarchy, and degree correlations. Phys Rev E. 2003;67(5):056104.

17. Al-Debei MM, Al-Lozi E, Papazafeiropoulou A. Why people keep coming back to facebook: explaining and predicting continuance participation from an extended theory of planned behaviour perspective. Decis Supp Syst. 2013;55(1):43-54.

18. Chen S-C, Yen DC, Hwang Ml. Factors influencing the continuance intention to the usage of web 2.0: an empirical study. Comput Human Behav. 2012;28(3):933-41.

19. Sun N, Rau PP-L, Ma L. Understanding lurkers in online communities: a literature review. Comput Human Behav. 2014;38:110-7.

20. Lőrincz L, Koltai J, Győr AF, Takács K. Collapse of an online social network: burning social capital to create it? Soc Netw. 2019;57:43-53.

21. Garcia, D., Mavrodiev, P., Schweitzer, F.: Social resilience in online communities: The autopsy of friendster. In: Proceedings of the First ACM Conference on Online Social Networks. COSN '13, pp. 39-50. ACM, New York, NY, USA (2013). https:// doi.org/10.1145/2512938.2512946.

22. Luqman A, Cao X, Ali A, Masood A, Yu L. Empirical investigation of facebook discontinues usage intentions based on sor paradigm. Comput Human Behav. 2017;70:544-55.

23. Yamakami, T: Towards understanding sns fatigue: exploration of social experience in the virtual world. In: 20127 th International Conference on Computing and Convergence Technology (ICCCT), pp. 203-207 (2012)

24. Xiong $X$, Jiang $\mathrm{D}, \mathrm{Wu} Y$, He L, Song H, Lv Z. Empirical analysis and modeling of the activity dilemmas in big social networks. IEEE Access. 2016;5:967-74.

25. Li D, Chen Z, Liu J. Analysis for behavioral economics in social networks: an altruism-based dynamic cooperation model. Int J Parallel Progr. 2019;47(4):686-708. https://doi.org/10.1007/s10766-018-0559-9.

26. White, J., Park, J.S., Kamhoua, C.A., Kwiat, K.A.: Game theoretic attack analysis in online social network (osn) services. In: Proceedings of the 2013 leee/acm International Conference on Advances in Social Networks Analysis and Mining, pp. 1012-1019 (2013). ACM

27. Yadati, N., Narayanam, R.: Game theoretic models for social network analysis. In: Proceedings of the 20th International Conference Companion on World Wide Web, pp. 291-292 (2011). ACM

28. Anderson E. Social Media Marketing: Game Theory and the Emergence of Collaboration. Berlin: Springer; 2010.

29. Fulk J, Flanagin AJ, Kalman ME, Monge PR, Ryan T. Connective and communal public goods in interactive communication systems. Commun Theory. 1996;6(1):60-87. https://doi.org/10.1111/j.1468-2885.1996.tb00120.x.

30. Leonardi PM, Huysman M, Steinfield C. Enterprise social media: definition, history, and prospects for the study of social technologies in organizations. J Comput Med Commun. 2013;19(1):1-19. https://doi.org/10.1111/jcc4.12029.

31. Osaka K, Toriumi F, Sugawara T. Effect of direct reciprocity and network structure on continuing prosperity of social networking services. Comput Soc Netw. 2017:4(1):2.

32. Toriumi, F., Yamamoto, H., Okada, I:: A belief in rewards accelerates cooperation on consumer-generated media. J Comput Soc Sci, 1-13

33. Leskovec, J., Krevl, A.: SNAP Datasets: Stanford Large Network Dataset Collection. http://snap.stanford.edu/data (2014)

34. Fechner GT, Howes DH, Boring EG. Elements of Psychophysics, vol. 1. New York: Holt; 1966.

35. Miura, Y., Toriumi, F., Sugawara, T.: Evolutionary learning model of social networking services with diminishing marginal utility. In: Companion Proceedings of the The Web Conference 2018. WWW'18, pp. 1323-1329. International World Wide Web Conferences Steering Committee, Republic and Canton of Geneva, Switzerland (2018). https://doi. org/10.1145/3184558.3191573.

36. Newman MEJ. Mixing patterns in networks. Phys Rev E. 2003;67:026126. https://doi.org/10.1103/PhysRevE.67.026126.

37. Piraveenan M, Prokopenko M, Zomaya AY. Local assortativeness in scale-free networks. Europhys Lett. 2008;84(2):28002. https://doi.org/10.1209/0295-5075/84/28002.

38. Aral S, Dhillon PS. Social influence maximization under empirical influence models. Nat Human Behav. 2018;2(6):375-82.

39. Assenza S, Gómez-Gardeñes J, Latora V. Enhancement of cooperation in highly clustered scale-free networks. Phys Rev E. 2008;78:017101. https://doi.org/10.1103/PhysRevE.78.017101.

\section{Publisher's Note}

Springer Nature remains neutral with regard to jurisdictional claims in published maps and institutional affiliations. 